\title{
Cogito ergo sum? - refocusing dementia ethics in a hypercognitive society
}

\author{
Desmond O’Neill
}

Ir J Psych Med 1997; 14(4): 121-123

The impact of dementia on individuals and society is enormous: one estimate of the cost of dementia care in the USA is over $\$ 100$ billion.' Dementia presents a unique challenge to healthcare ethics: never before has the healthcare system cared for so many with acquired, progressive mental disability. The implications of this disability are legion, and there has been a growing literature on the ethics of dementia care. ${ }^{2}$ Much of the focus to date has concentrated on competency to consent for medical treatment, and on end of life issues, particularly tube-feeding, do-not-resuscitate (DNR) orders and advance directives. While clearly important to clinical practice, this emphasis has diverted attention from other ethical issues: for example, the response of society to dementia, the impact of dementia on doctor-patient relationships and resource allocation for the care of dementia. It has also the quality of promoting an ethics of sickness and death rather than an ethics of health and hope.

Common principles to many ethics discourses are beneficence, non-maleficence, respect for autonomy and equality. Dementia ethics are special because of the impact of the disease on these concepts, particularly equality and autonomy. One of the main obstacles to equality for the patient with dementia is that we live in what Post refers to as a hypercognitive culture. This culture is the child of rationalism and capitalism, so that clarity of mind and economic productivity may seem to determine the value of human life. ${ }^{3}$ A disproportionate emphasis on cognitive function robs our ethical system of a basic equality among humans.

In dementia, we need to convert the dictum "I think, therefore I am" to "I will, feel and relate while disconnected by forgetfulness from my former self, but still, I am". The Judaeo-Christian ethic of caring has never accepted that the value of human life rests in reason and memory alone, but modern society has had some difficulty in accepting this loss of cognitive function. At a historical level, this trend is seen at its worst in the unethical research practices on patients with dementia ${ }^{4}$ or the murder of many thousands with dementia under the T-4 project in Nazi Germany.

Some commentators on dementia still hold an exclusionary position with regard to people with dementia: one philosopher has likened people with severe dementia to dogs, since they supposedly "lack capacities for hopes and fears, dreads and longings for their futures." One well known writer makes unfounded assumptions that patients in the last stages of dementia seem to have lost the capacity

Desmond O'Neill MD, FRCPI, Consultant in Geriatric Medicine, Age-Related Health Care, Adelaide and Meath Hospital Dublin incorporating the National Children's Hospital, Dublin 8, Ireland.

SUBMITTED; AUGUST 12, 1997. ACCEPTED: SEPTEMBER 29, 1997. to recognise or appreciate indignity, or to suffer from it. ${ }^{7}$ Even in everyday speech, it is not uncommon to hear remarks such as 'the patient is only a shell of his former self'.

These negative comments are not only repugnant to a moral solidarity with those suffering from dementia, but also contrast with many carers' experience that people with dementia have great sensitivity to attitudes and behaviour of those around them, and can derive pleasure from relationships, activity and creativity.

We need to develop our understanding of how to enable the positive aspects of living in dementia. There is a small but growing literature on the subjective experience of dementia among patients with dementia. ${ }^{8,9}$ Kitwood and Bredin have also made a useful contribution towards developing indicators of wellbeing in people with severe dementia. ${ }^{10}$ Some of this philosophy has crept into medical literature: among a sea of rating scales which assess mainly negative characteristics of dementia, it is encouraging to discover the Pleasant Event Schedule of Teri and Logsden. ${ }^{11}$ The key to an adequate ethics of dementia is full attention to the many ways of enhancing the non-cognitive aspects of wellbeing while not underestimating remaining capacities.

If this attention to the overall communication sensitivities and non-cognitive needs of the patient with dementia is neglected, the patient/physician relationship may be harmed and patient autonomy may be compromised..$^{12.13}$ In contrast to other illnesses, the patient may not initiate the consultation, or be a reluctant participant in the assessment/management process. While the cognitive traffic is disproportionately, but not entirely, skewed in the favour of the physician, the emotional and human elements are preserved. It is not uncommon for patients with dementia and their caregivers to complain of insensitive assessment whereby the physician did not interview the patient alone, and talked to the caregiver as if the patient was not in the room. It is a challenge to our humanity and professional skills to ensure that the patient feels that they are at the centre of a relationship where their fears and problems will be professionally and confidentially handled. At the very least, the patient should be interviewed on their own before any discussion with caregivers, and where feasible, permission should be sought for collateral history. A positive attitude will help us to resolve some of the conflicts with disclosure of the diagnosis: whereas carers are reluctant to share the diagnosis, ${ }^{14}$ older people state they would rather be informed of the diagnosis should they be affected. ${ }^{\text {Is }}$

The mental disability of dementia renders sufferers vulnerable in many ways, from elder abuse to the greatest ethical and moral challenge surrounding dementia, which is the lack of universal access to high quality assessment and management. It is very likely that far more suffering is caused to patients with dementia and their caregivers by 
undertreatment than by overtreatment. It is particularly worrying that a recent textbook of ethics in neurology should support discrimination against access to high technology life-support on the basis of age (ageism) and dementia (perhaps we could call this cognitivism) rather than on functional or physical status. ${ }^{16}$ Access to appropriate neuroradiology may be circumscribed due to these influences, many healthcare providers in the United Kingdom are refusing to provide funding for cholinesterase-inhibitor therapy, and patients with dementia and physical frailty are not offered community services to the same extent as their frail peers with normal cognitive function. ${ }^{17}$ Those who care for patients with dementia need to understand the potency of ageism and cognitivism in health and social services, and assume a role of advocacy to ensure adequate assessment and care facilities for their patients. For example, geriatricians in the United States have taken preliminary steps in formulating policy statements on allocation of medical resources. ${ }^{18}$

Caregivers are key collaborators in the development of ethical treatment of dementia..$^{19,20}$ Recognition of the enormous impact of dementing illness on caregivers should not blind us to the potential conflict between the needs of the patient and caregiver. Interventions for behavioural disturbance need to balance limitations to the patient and reduction of caregiver burden. ${ }^{21}$ Ideally social and environmental modification and the use of creative activities should be tried in the first instance. Similarly, respite care may cause upset and temporary worsening of cognitive state for the patient, but the long-term aim is to maximise the length of time which the patient can stay in their own home before institutionalisation.

As decision making capabilities decline with disease progression, early diagnosis and attention to care issues gives an opportunity to capitalise on residual competency and to plan for the future. Assessment of capacity to consent for medical treatment is an issue which has attracted much attention.22 Apart from an understanding of the difficulties of assessing competency, a knowledge of the growing literature in this field is important as it will help in understanding the uncertainties and prejudices which may underpin our decisions and help us to respond positively to them.

Relatives consistently choose a lesser intensity of treatment than patients would themselves, ${ }^{2:}$ nursing home residents state a majority preference for life-sustaining measures but discuss this with caregivers in only a minority of cases, ${ }^{24}$ healthcare workers display negative treatment attitudes to older patients (ageism), economic hardship encourages a lesser intensity of medical therapy by proxy decision makers ${ }^{25}$ and DNR orders tend to be made more commonly in groups who are discriminated against in other circumstances. ${ }^{26}$ These facts are not quoted to promote a charter of treatment of all diseases at a maximum intensity without regard to quality of life but rather to underline that families and healthcare professionals are probably more likely to undertreat than to overtreat diseases in later life and dementia.

As in other areas of intervention in dementia, careful assessment is the key to success. The interdisciplinary team need to gauge the patient's communication ability, cognitive and affective state: a psychosocial assessment of the patient and family should explore relationships, fears and understanding of patient and family. Several competency instruments have been developed for capacity to consent for medical treatment ${ }^{27}$ or making advance directives, ${ }^{28}$ but represent tentative steps in developing a knowledge base for the future. An assessment by a senior physician after appropriate assessment is still the standard measure of competency. It is increasingly clear that it is best to view competency as decision-specific: an excellent review on the subject is recommended. ${ }^{29}$ Research on physicians' consistency in assessing competency is inconclusive. Although one study suggested that agreement was not good when assessing patients with mild $\mathrm{AD}$, this was on the basis of viewing videos of patients with a mean Folstein MiniMental State Examination of $24::^{30}$ it is highly debatable whether this is more widely applicable.

In the first instance the patient's own views must be canvassed as there is often sufficient insight to make a decision. Unless the patient is a ward of court or under guardianship, the primary responsibility lies with the patient and the physician if the patient is adjudged incompetent. The views of the family should be canvassed along with appropriate psychosocial assessment: there is as yet little clear guidance for those without caregivers. ${ }^{31}$ Any refusal by the patient should be treated with respect. The main clinical priority is to detect and treat any condition which may influence this refusal, particularly depression and pain. In the absence of these factors it would be rare for a clinician to over-rule a refusal.

When a patient is no longer capable of participating in the decision-making process, clinicians need to confer with family to support the decision-making process, which usually hinges on issues of technical futility and quality of life issues. Technical futility may seem straightforward, but the impossible of today is the history of tomorrow (Marguerite Duras) and appropriate specialist referral is important. Quality of life is a difficult concept and in the event of dissonance between the family and the clinician, it may be wise to seek a second opinion: this is now the legal position in Ireland. ${ }^{32}$ The physician is not a neutral cipher in this process: he/she has a moral voice and in the absence of legal guardianship relates in the first instance to the patient rather than the carers. In some jurisdictions substituted judgement (an estimate of what the patient would have decided if competent and in the circumstances that currently obtain) is the standard of care (New York), and in others it the best interest principle (the treatment decision which is best serves the patient's current interests) (United Kingdom and Ireland).

A useful discussion on these approaches opts for a serious analysis of the experiential interests of the dementia patient, with elements of both approaches, but with a probable preference for best interests. ${ }^{3.3}$ Ideally the physician should respectfully recommend a course of action after conferring with the family rather than abandoning the decision to them. This has been described as an ethics of communication in which the physician is both a moderate autonomist and a moderate welfarist. ${ }^{34}$

Attitudes to advance directives vary between countries, races and cultures, and this may depend on many factors including the depth of the perceptions of over or undertreatment at the end of life. ${ }^{i 5}$ In the United States, advanced directives on resuscitation are now required as part of federal regulations to be discussed at the time of admission to healthcare facilities, including nursing homes. The difficulty of advance directives is illustrated by the commonest form of advance directive: "Don't put me in a nursing home when I'm old". Clearly it is very difficult for 


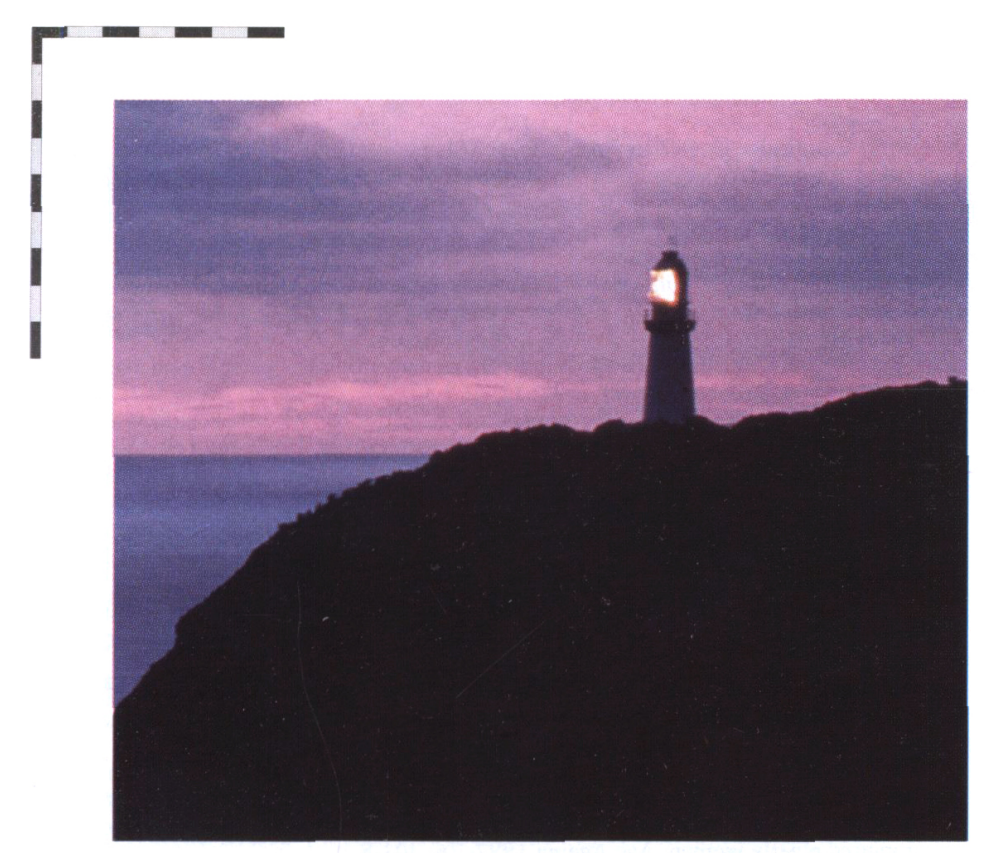

The Lundbeck Group, of Danish
origin, is a modern, research based pharmaceutical company. The Group is wholly owned by the Lundbeck Foundation, where all profits are re-invested in CNS research.

Many of the world's leading antidepressants have been developed by Lundbeck, including amitriptyline and nortriptyline. Lundbeck also navigated the development of lofepramine.

\section{A guiding light in the management of CNS illnesses}

Lundbeck was one of the first to introduce selective serotonin reuptake inhibitors (SSRIS), and citalopram (Cipramil), the most selective SSRI, is now market leader in eight European countries.

Here in Ireland, Lundbeck is market leader in depot anti-psychotics (Clopixol and Depixol) and its latest atypical anti-psychotic, Serdolect, will provide effective separation of therapeutic efficacy from extrapyramidal side effects (EPS).

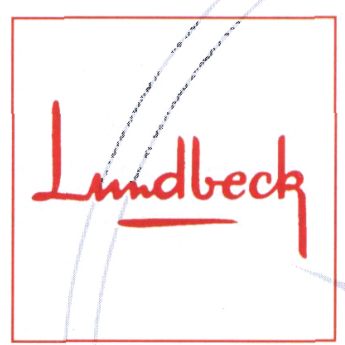

Pathfinders in CNS medicine since 1915.

Cipramil PA 776/1/2. Clopixol PA 1 15/5/1 3,4,5.7.9,10. Depixol PA 115/1/1,2,3,4,9,10. Serdolect PA 805/1/1 3,4,5. 
health providers and families to comply with this directive when living at home is no longer possible. Another difficulty is that advance directives are being promoted as a method of promoting healthcare cost savings. ${ }^{36}$ This will set alarm bells ringing for those who look after older people with disabling illness.

Of more relevance to end of life issues is an understanding of the phenomenology of death in dementia and a more finely developed expertise in palliative and hospice care of dementia. This type of approach has been described, ${ }^{37}$ and will help to change a passive approach of non-intervention into a positive one of palliative care. Weight loss and swallow disorders is one of the key areas and requires skillful handling. Once a full assessment has been carried out and other remediable factors treated such as depression or oral candidiasis, compensatory strategies such as semi-solid feeding can be considered. It is the author's experience that patients do not take kindly to modification of their fluid intake, and that free fluid intake and palliative care of the symptoms of aspiration may be more humane than modification and restriction of normal diet. Other alternatives such as feeding by percutaneous endoscopic gastrotomy (PEG) can raise ethical dilemmas, as much by the relative ease and safety of performing the procedure. The current state of medical knowledge leaves unanswered the question of whether routine tube feeding of demented patients maximises their interests: ${ }^{38}$ the benefits and disadvantages will have to be weighed in each individual case. For example, the stroke-related dysphagia of a patient with early vascular dementia and reasonably preserved function is very different to the anorexia of advanced Alzheimer's disease, and PEG feeding might be more clearly indicated in the former case.

A challenge for the future is how to heighten sensitivity among healthcare workers to the ethics of moral solidarity with those suffering from dementia. Despite increasing support from senior academic clinicians, ${ }^{39}$ ethics courses tend to have a low priority in medical schools, and those teaching are often not clinicians. While not devaluing the contribution of these teachers, students may sense that clinicians are not comfortable at the cutting edge of ethical debate. Research into ethical issues is limited and seems to have low priority in the United Kingdom and Ireland. Academic clinicians need to take the initiative to ensure that the imperatives-for ethical treatment are developed by clinicians rather than by the law, which is anethical and is rarely sensitive to the ethical nuances of clinical care.

\section{References}

1. Rice DP Fox PJ Max W et al. The economic burden of Alzheimer's disease care. Health Affairs $1993 ; 12$ : 164-76.

2. Jones RG. Ethical and legal jssues in the care of demented people. Rev Clin Gerontol 1997; 7: 147-62.

3. Post SG. The moral challenge of Alzheimer disease. Johns Hopkins University Press, 1995.

4. Karlawish JHT, Sachs G. Research on the cognitively impaired: lessons and warnings from the emergency research debate. J American Geriatr Soc 1997; 45: 474-81.

5. Muller-Hill B. Murderous science: elimination by scientific selection of Jews, Gypsies and orhers (trans. G Fraser) Oxford: Oxford University Press, 1988. 6. Brock DW. Life and death: philosophical essays in biomedical ethics. New
York: Cambridge University Press, 1993: 372.

7. Dworkin R. Life's dominion: an argument about abortion, euthanasia and individual freedom. New York: Vintage, 1993: 234.

8. Kitwood T. The experience of dementia. Aging and Mental Health 1997; 1: 13-22.

9. Bahro M, Silber E, Sunderland T. How do patients with Alzheimer's disease cope with their illness? - a clinical experience report. J American Geriatr Soc 1995; 43: 41-6

10. Kitwood T, Bredin K. Towards a theory of dementia care: personhood and well-being. Ageing and Society 1992; 12: 269-87.

11. Teri L, Logsdon R. Identifying pleasant activities for Alzheimer's disease patients: the Pleasant Event Schedule - AD. Gerontologist 1991; 31: 413-6.

12. Cassell CK, Jameton AL. Dementia in the elderly: an analysis of medical responsibility. Annals of Internal Medicine 1981; 94: 802-7.

13. American Academy of Neurology Ethics and Humanities Subcommittee. Ethical issues in the management of the demented patient. Neurology 1996; 46: 1180-3.

14. Maguire C, Kirby M, Coen R, Lawlor B, Coakley D, O'Neill D. Family members' attitudes toward telling the patient with Alzheimer's disease their diagnosis. BMJ 1996; 313: 529-30.

15. Holroyd S, Snustad DG, Chalfoux ZL. Attitudes of older adults on being told the diagnosis of Alzheimer's disease. J American Geriatr Soc 1996; 44: 400-3.

16. Bernat JL. Ethical issues in neurology. Butterworth-Heinemann, 1994.

17. Ely M, Brayne C, Huppert FA, O'Connor DW, Pollitt PA. Cognitive impairment: a challenge for community care. A comparison of the domiciliary service receipt of cognitively impaired and equally dependent physically impaired elderly women. Age Ageing 1997; 26: 301-8.

18. Rosenfeld KE, Pearlman RA. Allocating medical resources: recommendations for a professional response. J American Geriatr Soc 1997; 45: 886-8.

19. Norberg A. Ethics in the care of the elderly with dementia. In: Gillon R, ed. Principles of Health Care Ethics. Chichester: Wiley, 1994: 721-32.

20. Post SG, Whitehouse P. Fairhill guidelines on ethics and the care of people with Alzheimer disease. In: Post SG. The moral challenge of Alzheimer disease. Johns Hopkins University Press, 1995: 62-80.

21. Post SG. Behaviour control and Alzheimer disease in perspective. Alzheimer Dis and Rel Disord 1992; 6: 73-6.

22. Cassell CK, Hays JR, Lynn J. Alzheimer's: decisions in terminal care. Patient Care 1991; 25: 125-34.

23. Seckler AB, Meier DE, Mulvihill M, Cammer Paris BE. Substituted judgment: how reliable are proxy predictions? Ann Intern Med 1991; 115: 92-8.

24. O'Brien LA, Grisso JA, Maislin G et al. Nursing home residents' preferences for life-sustaining treatment. JAMA 1995; 274 : 1775-9.

25. Covinsky KE, Landefeld CS, Teno J, Connors AF, Dawson N, Youngner S et al. Is economic hardship on the families of the seriously ill associated with patient and surrogate care preferences? Arch Intern Med 1996; 156: 1737-41. 26. Wenger NS, Pearson ML, Desmond KA et al. Epidemiology of do-notresuscitate orders. Arch Intern Med 1996; 155: 2056-62.

27. Janofsky JS, McCarthy RJ, Folstein MF. The Hopkins Competency Assessment Test: a brief method for evaluating patients' capacity to give informed consent. Hosp Community Psychiatry 1992; 43: 132-6.

28. Molloy DW, Silberfield M, Darzins P, Guyatt GH, Singer PA, Rush B et al. Measuring capacity to complete an advance directive. J Am Geriatr Soc 1996; 44: $660-4$.

29. Glass KC, Silberfeld M. Determination of competence. In: Gauthier S ed. Clinical diagnosis and management of Alzheimer's disease. London: Martin Dunitz, 1996

30. Marson DC, McInturff B, Hawkins L, Bartolucci A, Harrell L. Journal Am Geriatr Soc 1997; 45: 453-7.

31. Miller TE, Coleman CH, Cugliari AM. Treatment decisions for patients without surrogates: rethinking policies for a vulnerable population. I Am Geriatr Soc 1997; 45: 369-74.

32. Lynch J. In the Matter of a Ward of Court. Dublin: High Court, 1996(May 5). 33. Berghmans R. Ethical hazards of the substituted judgement test in decision making concerning the end of life of dementia patients. Int J Geriatr Psychiatry $1997 ; 12: 283-7$

34. Pellegrino ED, Thomasma DC. For the patient's good: the restoration of beneficence in health care. Oxford: Oxford University Press, 1988.

35. Alemayehu E, Molloy DW, Guyatt GH, Singer J, Penington G et al. Variability in physician's decisions on caring for chronically ill elderly patients: an international study. Can Med Assoc J 1991; 144: 1133-8.

36. Teno J, Lynn J, Connors AF, Wenger N, Phillips RS, Alzola C et al. The illusion of end-of-life resource savings with advance directives. J Am Geriatr Soc 1997; 45: 513-8.

37. Volicer L, Rheaume Y, Brown ], Fabiszewski K, Brady R. Hospice approach to the treatment of patients with advanced dementia of the Alzheimer type.J Am Med Assoc 1986; 256: 2210-13.

38. Ackerman TF. The moral implications of medical uncertainty: tube feeding demented patients. J Am Geriatr Soc 1996; 44: 1265-7.

39. Weatherall DG. Teaching ethics to medical students. J Med Ethics 1995; 21 : $133-4$ 